\title{
Cifras del Retroceso: El Deterioro Relativo de la Tasa de Mortalidad Infantil de Argentina en la Segunda Mitad del Siglo XX
}

\author{
Data of a Regression: The Relative Deterioration of \\ the Infant Mortality Rate of Argentina in the Second \\ Half of the XX Century
}

José Carlos Escudero ${ }^{1}$

Cristina M. Massa ${ }^{2}$

${ }^{1}$ Médico. Magíster en Sociología, Universidad de Michigan, EE.UU. Profesor Titular de la Universidad Nacional de Luján y Universidad Nacional de La Plata, Argentina. escuderosalud@gmail.com

2 Profesora Superior de Geografía. Universidad de Buenos Aires. Magíster en Demografía Social, Universidad Nacional de Luján. Coordinadora del Programa de Análisis Demográfico, INDEC, Argentina.

cristinamassa@arnet.com.ar
RESUMEN Se compara la evolución histórica de la tasa de mortalidad infantil de Argentina con la de otros países americanos en la segunda mitad del siglo XX. También se compara la evolución histórica de la tasa de mortalidad infantil de la Ciudad Autónoma de Buenos Aires y la del Gran Buenos Aires con la de otras ciudades de América y del mundo. Esto muestra el deterioro relativo de la posición de Argentina con respecto a su nivel de mortalidad infantil.

PALABRAS CLAVE Mortalidad Infantil; Tendencias; Datos Demográficos.

\begin{abstract}
A comparison is made of the historical development of the infant mortality rate of Argentina with other american countries in the second half of the XX Century. $A$ comparison is also made of the historical evolution of the infant mortality rate of the city of Buenos Aires and its agglomeration with other cities of South America and of the world. The relative deterioration of the placing of Argentina in relation to its infant mortality rate is made clear.
\end{abstract}

KEY WORDS Infant Mortality; Trends; Demographic Data. 


\section{INTRODUCCIÓN}

La tasa de mortalidad infantil (TMI) mide específicamente el riesgo de muerte en los niños desde el nacimiento hasta que han cumplido un año, pero se ha convertido además en un indicador reduccionista del nivel sanitario general de las poblaciones, y en un sentido más amplio en indicador del buen funcionamiento y la equidad que existen en las mismas. La TMI es muy usada en el discurso político y popular, siendo más difundida que, por ejemplo, la esperanza de vida al nacer (EVN), cuya capacidad de resumen y exactitud en medir la fuerza de la mortalidad es mayor que la TMI, al ser un indicador preciso de la mortalidad general y no sólo de un sector muy vulnerable de la población. Sin embargo, hay que tener presente que la reducción de la TMI tiene fuerte incidencia en la EVN.

Esta popularidad de la TMI Ileva a que el poder político y sus divulgadores mediáticos presten mucha atención a su evolución en el tiempo, y a desear para ella el nivel más bajo posible; aunque los políticos no instrumenten las medidas para hacerlo y los divulgadores no denuncien su escasez o ausencia. De forma más general, debemos hacer notar que los adelantos sanitarios Ilevan legitimidad al poder político que los invoca, sean estos adelantos ciertos o falsos. El carácter de la salud como elemento central en el deseo de las poblaciones, y su capacidad de movilizar grandes capitales (se estima que el gasto mundial en salud oscila entre el $8 \%$ y el $10 \%$ del PBI mundial, en constante aumento), hace que políticos y divulgadores aplaudan disminuciones en la TMI sin tener en cuenta la calidad de los datos de ésta, ni efectuar comparaciones con otras áreas y países donde la situación es mejor; y sin hacer pública la gran dificultad política para mejorarla en un campo como la salud, donde existen intereses económicos tan poderosos.

La idea de que Argentina ha configurado un fracaso histórico extremo, un país del cual se esperaba un desarrollo promisorio hace un siglo, y que evolucionó hasta su actual tercermundización, se halla muy extendida en el pensamiento de las Ciencias Sociales y ha sido objeto de un intenso tratamiento académico, especialmente en los últimos años $(1,2)$. Polak (3) nos recuerda los comentarios de dos Premios Nobel de Economía: en 1942, Colin Clark pronosticó que en 1960 el Producto Bruto por habitante de Argentina iba a ser inferior solamente al de Estados Unidos, pero superior a todos los demás países; y en 1945 Paul Samuelson vaticinó para Argentina el mayor desarrollo económico mundial en las siguientes tres décadas.

El deterioro sanitario, en cambio, ha sido poco tratado $(4,5,6)$. El propósito de este artículo es medir este deterioro siguiendo la tendencia de la TMI en la Argentina en la segunda mitad del siglo XX, en comparación con la de otros países del continente americano. Queda claro que el deterioro del que hablamos es relativo: en términos absolutos la TMI argentina ha continuado descendiendo y, como no se cansan de repetir los funcionarios, es cada año sucesivo, con muy pocas excepciones (cuando coyunturalmente aumenta un poco), la más baja de la historia nacional. Esta visión panglossiana se desvanece cuando se compara la Argentina con otros países, en los que el descenso de la TMI ha sido mucho más importante y rápido.

Centraremos nuestro análisis en la segunda mitad del siglo XX, por la escasez de datos y por su baja calidad en tiempos anteriores, debido al subregistro a veces estructural y a veces deliberado de la TMI en muchos países. Como marco general podemos contextualizar la Argentina, a partir de su modernización y exitosa integración con el mercado mundial a fines del siglo XIX, como un país anómalo en términos demográficos y sanitarios: un receptor muy importante de inmigrantes, en su gran mayoría europeos, que fueron exitosamente integrados en gran parte mediante un sistema educativo estatal de excepcional calidad, y donde el bajo desempleo, el bajo costo de los alimentos y el desarrollo de un saneamiento eficaz en las ciudades producía un buen nivel sanitario, y consecuentemente una baja TMI para la época. Minujín y Kessler (7) han señalado que en la década del ' 30 del siglo XX, la Argentina tenía una TMI más baja que Italia o España, países de los que recibíamos el mayor número de inmigrantes (8). 


\section{LOS DATOS}

El análisis de la tendencia de la mortalidad infantil en los diferentes países se basa en una combinación de información proveniente de los registros de estadísticas vitales y de censos de población. Sin embargo, dicha información, contenida en las proyecciones de población de los distintos países, ha sido corregida atendiendo a las deficiencias que suelen presentar las mismas en cuanto a cobertura, especialmente en décadas pasadas (9).
También es necesario señalar que mientras en algunos países de América Latina el registro de defunciones infantiles es bastante completo, como en el caso de Argentina, Costa Rica, Chile, Cuba, México, Uruguay y Venezuela $(9,10)$, en otros países existen elevados porcentajes de subregistro o bien el registro de estadísticas vitales es sumamente deficiente, recurriéndose en ellos a estimaciones indirectas de la mortalidad infantil, teniendo en cuenta para ello la información que proveen preguntas específicas incluidas en los relevamientos censales o bien en encuestas demográficas (9).

Cuadro 1. TASAS DE MORTALIDAD INFANTIL POR PAÍS Y QUINQUENIO. ARGENTINA Y AMÉRICA LATINA, 1950-2005.

TASAS DE MORTALIDAD INFANTIL (por mil nacidos vivos)

\begin{tabular}{|c|c|c|c|c|c|c|c|c|c|c|c|}
\hline PAÍSES & 1950-1955 & $1955-1960$ & $1960-1965$ & $1965-1970$ & 1970-1975 & $1975-1980$ & 1980-1985 & $1985-1990$ & 1990-1995 & $1995-2000$ & $2000-2005$ \\
\hline América Latina & 127,7 & 114,2 & 102,1 & 92,3 & 81,7 & 69,8 & 57,5 & 47,6 & 39,2 & 33,0 & 28,0 \\
\hline Argentina & 65,9 & 60,4 & 59,7 & 57,4 & 48,1 & 39,1 & 32,2 & 27,1 & 24,4 & 21,8 & 15,0 \\
\hline Bolivia & 175,7 & 169,7 & 163,6 & 157,5 & 151,3 & 131,2 & 109,2 & 90,1 & 75,1 & 66,7 & 55,6 \\
\hline Brasil & 134,7 & 121,9 & 109,4 & 100,1 & 90,5 & 78,8 & 63,3 & 52,4 & 42,5 & 34,1 & 27,3 \\
\hline Chile & 120,3 & 118,3 & 109,0 & 89,2 & 68,6 & 45,2 & 23,7 & 18,4 & 14,1 & 11,5 & 8,0 \\
\hline Colombia & 123,2 & 105,3 & 92,1 & 82,2 & 73,0 & 56,7 & 48,4 & 41,4 & 35,2 & 30,0 & 25,6 \\
\hline Costa Rica & 93,8 & 87,7 & 81,3 & 67,7 & 52,5 & 30,4 & 19,2 & 17,4 & 14,5 & 11,8 & 10,5 \\
\hline Cuba & 80,6 & 69,9 & 59,4 & 49,7 & 38,5 & 22,5 & 17,0 & 12,9 & 10,0 & 7,5 & 7,3 \\
\hline Ecuador & 139,5 & 129,4 & 119,2 & 107,1 & 95,0 & 82,4 & 68,5 & 55,5 & 44,2 & 33,3 & 24,9 \\
\hline El Salvador & 151,1 & 137,0 & 122,7 & 110,3 & 105,0 & 95,0 & 77,0 & 54,0 & 40,2 & 32,0 & 26,4 \\
\hline Guatemala & 140,8 & 133,8 & 126,7 & 115,5 & 102,5 & 90,9 & 79,3 & 67,1 & 54,8 & 45,5 & 38,6 \\
\hline Haití & 219,6 & 193,5 & 176,2 & 165,2 & 152,2 & 139,2 & 122,1 & 100,1 & 74,1 & 66,1 & 59,1 \\
\hline Honduras & 169,3 & 153,9 & 135,5 & 119,0 & 103,7 & 81,0 & 65,0 & 53,0 & 43,0 & 35,0 & 31,2 \\
\hline México & 121,2 & 101,5 & 88,0 & 79,4 & 69,0 & 56,8 & 47,0 & 39,5 & 34,0 & 31,0 & 28,2 \\
\hline Nicaragua & 172,3 & 150,7 & 131,3 & 113,8 & 97,9 & 90,1 & 79,8 & 65,0 & 48,0 & 35,0 & 30,1 \\
\hline Panamá & 93,0 & 74,9 & 62,7 & 51,6 & 43,7 & 36,3 & 31,6 & 29,6 & 27,0 & 23,7 & 20,6 \\
\hline Paraguay & 73,4 & 69,7 & 62,3 & 58,6 & 53,1 & 51,0 & 48,9 & 46,7 & 43,3 & 39,2 & 37,0 \\
\hline Perú & 158,6 & 148,2 & 136,1 & 126,3 & 110,3 & 99,1 & 81,6 & 68,0 & 55,5 & 42,1 & 33,4 \\
\hline Rep. Dominicana & 149,4 & 132,2 & 117,5 & 105,0 & 93,5 & 84,3 & 62,5 & 54,1 & 46,6 & 440,0 & 34,4 \\
\hline Uruguay & 57,4 & 53,0 & 47,9 & 47,1 & 46,3 & 42,4 & 33,5 & 22,6 & 20,1 & 17,5 & 13,1 \\
\hline Venezuela & 106,4 & 89,0 & 72,8 & 59,5 & 48,7 & 39,3 & 33,6 & 26,9 & 23,2 & 20,7 & 17,5 \\
\hline $\begin{array}{l}\text { Número de países co } \\
\text { menor TMl que } \\
\text { Argentina }\end{array}$ & 1 & 1 & 2 & 3 & 3 & 3 & 4 & 5 & 5 & 5 & 4 \\
\hline
\end{tabular}


CUadro 2. TASAS DE MORTALIDAD INFANTIL POR PAÍS O ÁREA DE REGISTRO Y QUINQUENIO. ARGENTINA Y EL CARIBE, 1950-2005.

TASAS DE MORTALIDAD INFANTIL

(por mil nacidos vivos)



\begin{tabular}{|c|c|c|c|c|c|c|c|c|c|c|c|}
\hline Antillas Holandesas & 69,0 & 51,0 & 42,0 & 35,0 & 28,0 & 22,0 & 18,0 & 17,0 & 16,3 & 14,2 & 12,6 \\
\hline Argentina & 65,9 & 60,4 & 59,7 & 57,4 & 48,1 & 39,1 & 32,2 & 27,1 & 24,4 & 21,8 & 15,0 \\
\hline Bahamas & 78,8 & 56,3 & 48,3 & 41,2 & 38,2 & 35,4 & 29,6 & 23,1 & 20,4 & 19,1 & 17,7 \\
\hline Barbados & 132,0 & 87,0 & 61,0 & 46,0 & 33,0 & 27,0 & 16,9 & 15,2 & 14,0 & 12,4 & 10,9 \\
\hline Belice & 88,0 & 78,0 & 69,0 & 60,0 & 52,0 & 45,0 & 39,3 & 35,9 & 34,6 & 33,3 & 31,1 \\
\hline Guadalupe (*) & 79,5 & 60,0 & 48,9 & 44,9 & 38,5 & 31,9 & 24,7 & 22,0 & 9,2 & 8,3 & 7,4 \\
\hline Guyana & 119,0 & 105,0 & 95,0 & 82,0 & 79,0 & 67,0 & 69,3 & 65,6 & 56,7 & 55,6 & 51,2 \\
\hline Guyana Francesa & 103,4 & 89,1 & 73,1 & 51,4 & 45,9 & 42,9 & 32,0 & 25,0 & 19,9 & 16,4 & 14,3 \\
\hline Jamaica & 91,9 & 78,3 & 61,4 & 51,6 & 45,0 & 37,0 & 30,5 & 27,0 & 24,3 & 21,9 & 19,9 \\
\hline Martinica (*) & 64,7 & 55,7 & 47,7 & 42,3 & 34,7 & 21,9 & 14,0 & 10,1 & 7,6 & 7,0 & 6,8 \\
\hline Puerto Rico (**) & 63,4 & 51,4 & 44,8 & 33,3 & 25,3 & 19,7 & 17,2 & 13,8 & 11,6 & 11,0 & 10,3 \\
\hline Santa Lucía & 114,6 & 105,3 & 81,1 & 47,7 & 39,1 & 29,3 & 22,7 & 20,1 & 16,9 & 16,9 & 14,8 \\
\hline Surinam & 89,2 & 76,2 & 63,5 & 54,6 & 48,8 & 44,0 & 40,3 & 36,1 & 33,4 & 29,1 & 25,7 \\
\hline Trinidad y Tobago & 76,0 & 63,0 & 48,0 & 45,6 & 41,1 & 32,0 & 25,3 & 19,7 & 16,3 & 15,1 & 14,1 \\
\hline $\begin{array}{l}\text { Número de países y } \\
\text { áreas de registro con } \\
\text { menor TMl que } \\
\text { Argentina }\end{array}$ & 2 & 5 & 6 & 11 & 10 & 9 & 10 & 10 & 10 & 9 & 8 \\
\hline
\end{tabular}

El análisis de la evolución de la TMI, por quinquenios, para el período 1950-2005, que se presenta en los Cuadros 1 y 2 , se realiza a partir de los resultados de las Proyecciones de Población vigentes en cada país, difundidas por el Centro Latinoamericano de Demografía (11) $y$, en el caso particular de los datos de Argentina, por el INDEC (12). Como estos datos corresponden al valor estimado de TMI en cada quinquenio del período histórico de la proyección (con excepción del lapso 2000-05), son comparativamente adecuados y contemplan por su naturaleza ajustes por corrección de la omisión en el registro de los hechos vitales, que como se sabe afecta más a las defunciones infantiles que a los nacimientos.

Entre los países de América Latina (Cuadro 1), la Argentina se ubica en el quinquenio 1950-55 en segundo lugar, siendo superada exclusivamente por Uruguay. Esta posición en el ranking de países de América Latina se sostiene hasta que en 1960-65 la Argentina pasa al tercer lugar como consecuencia del nivel de TMI alcanzado por Cuba (a).

En el quinquenio 1965-70, la Argentina vuelve a perder un lugar en el ranking al pasar al cuarto puesto, superada ahora por Uruguay, Cuba y Panamá. Con posterioridad, Argentina mantiene su cuarto puesto hasta fines de la década del '70. Sin embargo, se producen cambios entre los países con menores niveles de TMI, dado que Argentina se ubica por ese entonces por debajo de Cuba, Costa Rica y Panamá, dado que Uruguay no experimenta reducciones importantes durante este período y Costa Rica logra avances considerables a lo largo de la década (b).

En 1980-85, la Argentina pierde un lugar más (pasa al quinto puesto) al ser superada 
por Chile (c). En el período siguiente (1985-90), la Argentina se ubica en sexto lugar perdiendo otro puesto en el ranking, al alcanzar Venezuela niveles inferiores al de nuestro país. En este quinquenio, Uruguay logra ganancias en la mortalidad infantil que lo colocan en mejor posición respecto de Argentina, mientras Panamá experimenta un leve retroceso.

Entre 1990 y 2000, Argentina mantiene la sexta posición, período durante el cual se destaca el posicionamiento de Chile en el contexto de América Latina como consecuencia de la notable reducción de la TMI.

En el último período (2000-05), Argentina recupera una posición al ubicarse nuevamente en quinto lugar por el retroceso de Venezuela (puesto que había sido perdido 15 años antes), y se localiza por debajo de Cuba, Chile, Costa Rica y Uruguay. Cabe mencionar de todos modos que aún con esa posición en el ranking de América Latina, la TMI de Argentina duplica holgadamente el valor alcanzado por Cuba.

El deterioro de la TMI argentina ha tenido otras facetas. Habitualmente solemos comparar nuestra TMI con otros países latinoamericanos; eventualmente con EEUU y Canadá, y casi nunca con una serie de pequeños países americanos y áreas de registro que en general no hablan español, cuya invisibilidad para nosotros puede deberse a su pequeñez y a la raza negra de muchos de sus habitantes, cosa que incomoda al siempre existente racismo argentino. Pues bien, el retroceso argentino se evidencia también ante ellos. Es por eso que en el Cuadro 2 comparamos la TMI de Argentina con pequeños países y áreas de registro del Caribe, que tienen una población total de aproximadamente 11 millones de habitantes.

Replicando el análisis precedente para el contexto caribeño (d), la situación de Argentina es todavía más preocupante. De todos modos recordemos que dentro del contexto latinoamericano, la Argentina se ha caracterizado por presentar una buena cobertura de registro de hechos vitales, que no suele ser la norma en el resto de los países de América Latina, tal como se mencionó precedentemente. En consecuencia, si bien algunos datos deben ser tomados con el debido recaudo, dado que en el presente análisis se utiliza información proveniente de proyecciones de población, se estima que los datos implícitos en las mismas (TMI) han sido corregidos adecuadamente atendiendo a posibles problemas de cobertura.

A comienzos del período en estudio (1950-55), Argentina se ubica en un tercer puesto, presentando niveles superiores de TMI a Puerto Rico y Martinica (Cuadro 2). En el quinquenio siguiente (1955-60), el nivel de la mortalidad infantil de Argentina es superado además por las Antillas Holandesas, Bahamas y Guadalupe, quedando nuestro país en sexto lugar. Pero en el quinquenio 1960-65 pasa al séptimo lugar, como consecuencia del nivel de TMI alcanzado por Trinidad y Tobago.

En 1965-70, Argentina experimenta un deterioro notable en el contexto de los países del Caribe, pues desciende al duodécimo lugar, dado que Barbados, Santa Lucía, Guyana Francesa, Jamaica y Surinam presentan valores de TMI inferiores al de nuestro país.

A partir de comienzos de la década del '70, Argentina comienza a recuperar posiciones, pasando al undécimo lugar en 1970-75 y al décimo en 1975-80. Con posterioridad a un leve descenso experimentado entre 1980 y 1990, período durante el cual Argentina retrocede al undécimo lugar, entre 1995 y 2005 nuestro país recupera un lugar en cada uno de los quinquenios.

Lo señalado precedentemente permite apreciar que en el período (2000-05), Argentina se ubica en el noveno lugar si se la compara con estos países y áreas de registro del Caribe, luego de experimentar un marcado retroceso durante el período en estudio, presentando valores de mortalidad infantil que duplican los alcanzados por Martinica y Guadalupe.

Si consideramos otros países de América, como Estados Unidos y Canadá, obviamente la situación es desfavorable para Argentina. En el quinquenio 1950-55 las TMl de Argentina, Canadá y Estados Unidos era respectivamente de 65,9 por mil (12), 38,5 por mil (13) (e) y 29,2 por mil (14). En el quinquenio 2000-05 estas tasas habían descendido respectivamente a 15,0 por mil (12), 5,3 por mil (15) y 7,0 por mil (15); con un porcentaje de disminución respectivo de $77 \%$, $86 \%$ y $76 \%$. Esto cuestionaría la sabiduría convencional que postula mayores reducciones de la TMI para aquellos países cuyas tasas iniciales son mayores. 
La discusión habitual sobre el nivel de la TMI de Argentina no tiene en cuenta las grandes reducciones de dicha tasa que han ocurrido en las últimas décadas en otros países del mundo, cuya situación al inicio del período de comparación era a veces peor que la nuestra. En 1960, la TMI de Singapur y República de Corea eran de 35 por mil y 90 por mil respectivamente, comparado con una TMI para Argentina de 59,7 por mil (12). Para el año 2000 los dos primeros países habían disminuido respectivamente a 3 por mil y 5 por mil $(15,16)$, mientras que la tasa de Argentina era de 15 por mil (12).

\section{MORTALIDAD EN CIUDAD DE BUENOS AIRES Y EN OTRAS CIUDADES DEL MUNDO}

Resulta instructivo comparar la tendencia de la TMI en la Ciudad de Buenos Aires (dato de fácil hallazgo, ya que Buenos Aires ha configurado siempre, en términos estadísticos, un área de registro comparable a una provincia) con la de otras ciudades de América y del mundo.

El Cuadro 3 compara la Ciudad de Buenos Aires con otras ciudades del mundo que a comienzos del siglo XX tenían una TMI más alta. Como los datos de estas ciudades hacen referencia al aglomerado urbano en su conjunto, es decir al continuo urbano que se extiende más allá de los límites administrativos de la ciudad capital del país, para los fines comparativos se considerará al aglomerado Gran Buenos Aires, que incluye un área de registro aparte, conformada por los denominados Partidos del Gran Buenos Aires (f), que se suman a la Ciudad de Buenos Aires para efectuar comparaciones.

El Gran Buenos Aires tuvo a partir de los años cincuenta, un crecimiento explosivo de población que hoy triplica a Ciudad de Buenos Aires, y su nivel de vida, y en términos socioeconómicos su acceso a los servicios de salud es

Cuadro 3. TENDENCIA DE LAS TASAS DE MORTALIDAD INFANTIL EN LA CIUDAD DE BUENOS AIRES, EN EL GRAN BUENOS AIRES Y EN AGLOMERADOS URBANOS DE OTROS PAÍSES, 1900-2000.




Cuadro 4. ESTIMACIÓN DE LAS DEFUNCIONES INFANTILES ANUALES QUE SE HUBIERAN EVITADO SI SE HUBIESEN ALCANZADO LAS TMI DE PAÍSES Y ÁREAS DE REGISTRO DE AMÉRICA LATINA Y EL CARIBE CON TASAS INFERIORES, 2000-2005.

\begin{tabular}{|c|c|c|c|c|}
\hline PAÍSES (*) & $\begin{array}{c}\text { TMI } \\
(2000-2005)\end{array}$ & $\begin{array}{l}\text { Total de defunciones } \\
\text { infantiles anuales } \\
\text { estimadas para Argentina } \\
\text { con la TMl de cada país }\end{array}$ & $\begin{array}{l}\text { Total de defunciones } \\
\text { infantiles que se } \\
\text { hubieran evitado } \\
\text { anualmente en Argentina }\end{array}$ & $\begin{array}{l}\text { Porcentaje estimado de } \\
\text { defunciones que se } \\
\text { hubieran evitado }\end{array}$ \\
\hline Martinica & 6,8 & 4.618 & 5.595 & 54,8 \\
\hline Cuba & 7,3 & 4.957 & 5.256 & 51,5 \\
\hline Guadalupe & 7,4 & 5.025 & 5.188 & 50,8 \\
\hline Chile & 8,0 & 5.433 & 4.780 & 46,8 \\
\hline Puerto Rico & 10,3 & 6.995 & 3.218 & 31,5 \\
\hline Costa Rica & 10,5 & 7.130 & 3.083 & 30,2 \\
\hline Barbados & 10,9 & 7.402 & 2.811 & 27,5 \\
\hline Antillas Holandesas & 12,6 & 8.557 & 1.656 & 16,2 \\
\hline Uruguay & 13,1 & 8.896 & 1.317 & 12,9 \\
\hline Trinidad y Tobago & 14,1 & 9.575 & 638 & 6,2 \\
\hline Guyana Francesa & 14,3 & 9.711 & 502 & 4,9 \\
\hline Santa Lucía & 14,8 & 10.051 & 162 & 1,6 \\
\hline Argentina & 15,0 & 10.213 & 0 & 0,0 \\
\hline
\end{tabular}

Nota: (*) Países ordenados en forma ascendente de acuerdo con la TMI del quinquenio 2000-2005

apreciablemente inferior al de la Ciudad. Es por esto que hemos calculado por separado la TMI de Ciudad de Buenos Aires, de los partidos del Gran Buenos Aires y del denominado Gran Buenos Aires, conformado por la sumatoria de los dos anteriores (Cuadro 3).

\section{UNA REFLEXIÓN}

Un inconveniente de trabajar con "tasas" es que se enmascara el hecho terrible de la muerte de personas concretas. Es por ello que en el Cuadro 4 se contabilizan, a partir del promedio anual de nacimientos ocurridos en Argentina en el quinquenio 2000-05, y de las TMI de los países que en ese período presentan niveles inferiores de TMI que Argentina, cuántas defunciones de menores de un año se hubiesen evitado si Argentina presentara esos valores de TMI.

En el mencionado Cuadro se presenta el total de defunciones infantiles que hubieran ocurrido en Argentina si el volumen anual de nacimientos de nuestro país hubiera estado expuesto a los niveles de mortalidad infantil de los países de América Latina y Caribe que cuentan con una TMI más baja. Además se incluye el volumen de defunciones de menores de un año que se hubieran evitado en nuestro país si Argentina presentara los valores de TMI de esos países. 


\section{NOTAS FINALES}

a. Cuba logra reducir la TMI entre $1950-55$ y 1960-65 de 80,6 a 59,4 por mil, es decir un $26 \%$.

b. Costa Rica logra una reducción del $42 \%$ de la TMI de 1975-80 respecto de la de 1970-75.

c. Chile reduce el nivel de la mortalidad infantil en un $48 \%$ entre $1975-80$ y $1980-85$. d. Se aclara que se sigue el criterio utilizado por CEPAL-CELADE para la identificación del contexto caribeño.

e. Datos del año 1951.

f. Se trata de 31 partidos de la provincia de Buenos Aires que integran total o parcialmente el aglomerado urbano.

\section{REFERENCIAS BIBLIOGRÁFICAS}

1. Cortes Conde R. La economía política de Argentina en el siglo XX. Buenos Aires: Edhasa; 2005.

2. Gerchunoff P, Llach L. El ciclo de la ilusión y el desencanto: un siglo de política económica argentina. Buenos Aires: Ariel; 2005.

3. Polak F. Un viejo diccionario español: la tontería del desarrollo económico. Buenos Aires: Corregidor; 2005.

4. Escudero JC. La salud al final de siglo. Sociedad. 1999;15:55-63.

5. Veronelli JC, Veronelli Correch M. Los orígenes institucionales de la Salud Pública Argentina. Buenos Aires: OPS; 2004.

6. Moyano G, Escudero JC. La salud en Argentina (en manos de quién). En: Derecho a la salud, situación en países de América Latina. Bogotá: Plataforma Interamericana de Derechos Humanos, Democracia y Desarrollo (PIDHDD), Asociación Latinoamericana de Medicina Social (ALAMES); 2005.

7. Minujín A, Kessler G. La nueva pobreza en la Argentina. Buenos Aires: Temas de Hoy; 1995.
8. Lattes A. El crecimiento de la población y sus componentes demográficos entre 1870 y 1970. En: La Población de Argentina. Buenos Aires: CICRED; 1975. p. 29-66.

9. Chackiel J. Mortality in Latin America. En: Health and Mortality Issues of Global Concern. Population and Family Study Centre, Flemish Scientific Institute. Bruselas: United Nations; 1997. p.132-157.

10. Chackiel, J. La dinámica demográfica en América Latina. Santiago de Chile: CEPAL-CELADE; 2004 (Población y Desarrollo N 52).

11. CEPAL-CELADE. América Latina y Caribe: Estimaciones y Proyecciones de Población 19502050. Santiago de Chile: CEPAL-CELADE; 2004. (Boletín Demográfico No 73).

12. INDEC-CEPAL-CELADE. Estimaciones y proyecciones nacionales de población por sexo y edad. 1950-2015. Buenos Aires, INDEC; 2004 (Serie Análisis Demográfico № 30).

13. United Nations. Economic Commission for Europe. United Nations Population Fund-Fertility and Family Surveys. Standard Country Report Canada. New York, Ginebra: UN; 1999. 
14. Bureau of the Census. Statistical Abstract of the United States. Washington: Department of Commerce; 1980-83, 1994.

15. United Nations. Demographic Yearbook 2002. New York: UN; 2002. (vol 54).

16. Krueger AO. WTO Ministerial Meeting, Hong Kong. Address to the Plenary Session [en línea] 14 de diciembre de 2005 [fecha de acceso 20 de febrero de 2006] URL disponible en: http://www.imf.org/external/np/speeches/2005/121405.htm

17. Mazzeo V. Mortalidad infantil en la Ciudad de Buenos Aires: 1856-1986. Buenos Aires: CEDAL; 1993.

18. Ministerio de Salud. Dirección de Estadística e Información de Salud. Indicadores de salud
seleccionados-República Argentina. Años 1980 a 1999. Buenos Aires: Ministerio de Salud; 2001.

19. Ministerio de Salud. Dirección de Estadística e Información de Salud. Estadísticas VitalesInformación Básica Año 2000. Buenos Aires: Ministerio de Salud; 2001.

20. O'Brien E, Benedict L, compiladores. Municipal Reference Collection. Chicago: Chicago Public Library; 1999.

21. Lee $\mathrm{CH}$. Regional inequalities in Infant Mortality in Britain 1861-1971, Patterns and Hypotheses. Population Studies. 1991;45(1):55-65.

22. Ministerio de Salud Pública de Cuba. Anuario Estadístico. La Habana: MSPC; 1998.

\section{FORMA DE CITAR}

Escudero JC, Massa CM. Cifras del Retroceso: El Deterioro Relativo de la Tasa de Mortalidad Infantil de Argentina en la Segunda Mitad del Siglo XX. Salud Colectiva. 2006;3(3):249-257.

Recibido el 27 de marzo de 2006

Versión final presentada el 26 de junio de 2006

Aprobado el 2 de agosto de 2006 